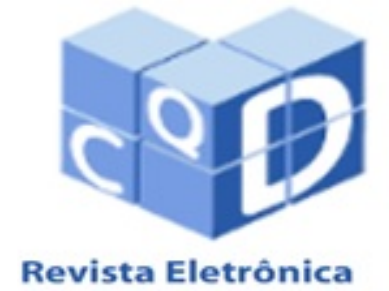

Paulista de Matemática

ISSN 2316-9664

Volume 7, dez. 2016

Edição ERMAC

Helen Gomes da Silva

UNESP - FFC

helen-277@hotmail.com

Hércules de Araujo Feitosa

UNESP - FC

haf@fc.unesp.br

\section{Lógica da Verdade Pragmática apresentada num sistema dedutivo de Tableaux}

Logic of Pragmatic Truth presented in a Tableaux deductive system

\begin{abstract}
Resumo
Inspirado na noção de quase-verdade de Newton da Costa, Silvestrini (2011) apresentou uma definição de verdade pragmática via satisfação pragmática e, no mesmo trabalho, introduziu a Lógica da Verdade Pragmática (LPT) em um sistema axiomático. LPT é uma lógica paraconsistente e trivalente. Posteriormente, Feitosa e Silvestrini (2016) apresentaram algumas alterações no conjunto de axiomas de LPT e deram uma demonstração de adequação segundo a semântica matricial de LPT. Neste artigo, introduzimos um sistema de tableaux, neste caso analítico, para a Lógica da Verdade Pragmática (LPT) e verificamos que os tableaux introduzidos são característicos de LPT.
\end{abstract}

Palavras-chave: Quase-verdade. Verdade Pragmática. Lógica Paraconsistente. Lógica Trivalente. Tableaux Analíticos.

\begin{abstract}
Inspired by notion of quasi-truth proposed by Newton da Costa, Silvestrini (2011) presented a definition of pragmatic truth via pragmatic satisfaction and, in the same paper, he introduced the Logic of Pragmatic Truth (LPT), in an axiomatic system. LPT is a paraconsistent and trivalent logic. Later, Feitosa and Silvestrini (2016) presented some changes in the axioms of LPT and a proof of adequacy for LPT in accordance to his matrix semantic. In this article, we introduce an analytical tableaux system for LPT and verify that these tableaux are characteristic for LPT.
\end{abstract}

Keywords: Quasi-truth. Pragmatic Truth. Paraconsistent logic. Trivalent Logica. Analytiuc Tableaux. 


\section{Introdução}

Motivados pelos trabalhos dos filósofos pragmáticos William James e Carl S. Peirce, Costa; Béziau e Bueno (1998) desenvolveram uma teoria da verdade, a qual denominaram de quaseverdade ou verdade pragmática.

Nesta tradição, a verdade está inserida e considerada no contexto das ciências empíricas, quando pode ocorrer que duas teorias que explicam o mesmo fenômeno sejam conflitantes. Se isto ocorre, a teoria da quase-verdade indica que estas proposições contraditórias podem ser ambas quase verdadeiras.

Costa; Béziau e Bueno (1998), ao formalizarem a quase verdade, consideraram que as estruturas nas quais seriam interpretadas as sentenças da linguagem objeto deveria ser parcial, em contraste com alguma estrutura total, na qual funda-se a concepção de verdade de Tarski.

Numa estrutura parcial, a pertinência ou não de uma dada n-upla do domínio não precisa estar sempre definida. As fórmulas atômicas são interpretadas por meio de uma relação parcial $R$, a qual é definida como um terna ordenada de conjuntos le $\left\langle R_{+}, R_{-}, R_{u}\right\rangle$. A componente $R_{+}$ representa o conjunto de n-uplas que satisfazem a relação $R, R_{-}$representa o conjunto de n-uplas que não satisfazem $R$, e $R_{u}$ representa o conjunto de n-uplas cuja pertinência é indeterminada. Não sabemos se os elementos de $R_{u}$ satisfazem ou não a relação $R$.

Diante disso, a relação parcial caracteriza o nosso conhecimento parcial do mundo, visto que não conhecemos tudo sobre determinada teoria e a componente $R_{u}$ representa a incompletude do que é sabido num dado momento.

Como desdobramento, Newton da Costa (1999) apresentou uma lógica modal para formalizar a sua concepção de quase-verdade, a qual foi desenvolvida através de dois sistemas axiomáticos modais, com inter-relações entre eles.

Em 2011, Silvestrini propôs uma nova definição para a quase-verdade via o conceito de satisfação pragmática e apresentou uma lógica paraconsistente de primeira ordem, denotada por LPT1 e apresentada como um sistema axiomático, para esta nova noção de verdade pragmática.

Neste trabalho, tratamos do fragmento proposicional da LPT1, isto é, a Lógica da Verdade Pragmática (LPT), no inglês Logic of Pragmatic Truth. Contudo, usamos a apresentação de Feitosa e Silvestrini (2016), que traz algumas modificações no conjunto de axiomas da LPT.

Iniciamos com a apresentação da Lógica da Verdade Pragmática conforme os textos mencionados. Descrevemos a LPT com o seu conjunto de axiomas e a sua semântica de matrizes trivalentes.

A seguir, tratamos dos tableaux e apresentamos o método para a lógica proposicional clássica.

Os sistemas de tableaux analíticos foram muito bem investigados por Smullyan (1968), inspirados nos métodos finitários de Gentzen (1935). Tal sistema dedutivo é considerado como um método de refutação, uma vez que para provar a validade de uma fórmula, supõe-se que a mesma é falsa e, então, busca-se uma situação que corrobora a falsidade da fórmula em teste. Se não há a situação de falsidade, então conclui-se que a fórmula inicial é válida.

Ademais, o tableau é caracterizado como um algoritmo em forma de árvore, que apresenta uma figura no processo de demonstração.

Este caminho caracteriza o método ou árvore de refutação.

Conforme Smullyan (1968), o tableau é analítico ao admitir o princípio das subfórmulas. No desenvolvimento da árvore de demonstração de uma fórmula, segundo os tableaux, ocorrem a cada passo apenas subfórmulas das fórmulas envolvidas. Assim, o procedimento envolve apenas subfórmulas da fórmula inicial. 
Se não conseguimos provar a validade de uma fórmula, o tableau indica de modo direto uma situação que faz a fórmula não válida, ou seja, apresenta um contra-exemplo.

Dando sequência, apresentamos um sistema dedutivo de tableaux analíticos para a LPT. Para mostrarmos que os tableaux introduzidos são característicos de LPT, mostramos que todas as deduções possíveis em LPT têm seus respectivos tableaux fechados no novo sistema. Por outro lado, verificamos que todas as deduções corretas nos tableaux preservam a validade na semântica matricial de LPT.

\section{A lógica da verdade pragmática}

Ao formalizar o conceito de verdade pragmática, Silvestrini (2011) observou que a lógica proposicional subjacente seria uma lógica paraconsistente. Esta lógica foi nomeada de lógica da verdade pragmática e denotada por LPT.

LPT admite a seguinte semântica trivalente associada com a relação $\left\langle R_{+}, R_{-}, R_{u}\right\rangle$ : valor 1 para sentenças verdadeiras, valor 0 para sentenças falsas, e valor $\frac{1}{2}$ para as sentenças indeterminadas.

A linguagem proposicional de LPT é $L=(\neg, \wedge, \rightarrow)$, em que os operadores $\neg, \wedge, \rightarrow$ representam, respectivamente, as noções de negação, conjunção e condicional.

As interpretações em matrizes desses operadores são as seguintes:

\begin{tabular}{|c||c|c|c|}
\hline$\rightarrow$ & 0 & $\frac{1}{2}$ & 1 \\
\hline \hline 0 & 1 & 1 & 1 \\
\hline$\frac{1}{2}$ & 0 & 1 & 1 \\
\hline 1 & 0 & 1 & 1 \\
\hline
\end{tabular}

\begin{tabular}{|c||c|c|c|}
\hline$\wedge$ & 0 & $\frac{1}{2}$ & 1 \\
\hline \hline 0 & 0 & 0 & 0 \\
\hline$\frac{1}{2}$ & 0 & $\frac{1}{2}$ & $\frac{1}{2}$ \\
\hline 1 & 0 & $\frac{1}{2}$ & 1 \\
\hline
\end{tabular}

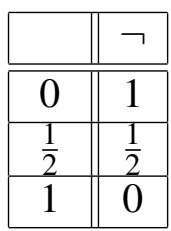

Além desses operadores básicos, temos os seguintes operadores e constantes de LPT:

Disjunção: $\varphi \vee \psi={ }_{\text {def }} \neg(\neg \varphi \wedge \neg \psi)$

Top: $\top{ }_{\text {def }} \varphi \rightarrow \varphi$

Botton: $\perp={ }_{\text {def }} \neg \top$

Negação clássica: $\sim \varphi={ }_{\text {def }} \varphi \rightarrow \perp$

Consistência: $\circ \varphi={ }_{\text {def }} \sim(\varphi \wedge \neg \varphi)$

Bicondicional: $\varphi \leftrightarrow \psi={ }_{\text {def }}(\varphi \rightarrow \psi) \wedge(\psi \rightarrow \varphi)$.

As suas interpretações são dados pelas seguintes tabelas: 


\begin{tabular}{|c||c|c|c|}
\hline$\vee$ & 0 & $\frac{1}{2}$ & 1 \\
\hline \hline 0 & 0 & $\frac{1}{2}$ & 1 \\
\hline$\frac{1}{2}$ & $\frac{1}{2}$ & $\frac{1}{2}$ & 1 \\
\hline 1 & 1 & 1 & 1 \\
\hline
\end{tabular}

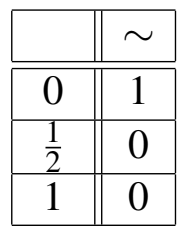

\begin{tabular}{|c||c|}
\hline & $\circ$ \\
\hline \hline 0 & 1 \\
\hline$\frac{1}{2}$ & 0 \\
\hline 1 & 1 \\
\hline
\end{tabular}

\begin{tabular}{|c||c|c|c|}
\hline$\leftrightarrow$ & 0 & $\frac{1}{2}$ & 1 \\
\hline \hline 0 & 1 & 0 & 0 \\
\hline$\frac{1}{2}$ & 0 & 1 & 1 \\
\hline 1 & 0 & 1 & 1 \\
\hline
\end{tabular}

\begin{tabular}{|c||c|}
\hline & $\top$ \\
\hline \hline 0 & 1 \\
\hline$\frac{1}{2}$ & 1 \\
\hline 1 & 1 \\
\hline
\end{tabular}

\begin{tabular}{|c||c|}
\hline & $\perp$ \\
\hline \hline 0 & 0 \\
\hline$\frac{1}{2}$ & 0 \\
\hline 1 & 0 \\
\hline
\end{tabular}

Segue da definição da disjunção $\vee$ e das tabelas de $\wedge$ e $\vee$, que valem na LPT as leis de De Morgan $\neg(\varphi \wedge \psi) \leftrightarrow(\neg \varphi \vee \neg \psi) \mathrm{e} \neg(\varphi \vee \psi) \leftrightarrow(\neg \varphi \wedge \neg \psi)$.

A semântica matricial de LPT é:

$$
\mathscr{M}_{L P T}=\left(\left\{0, \frac{1}{2}, 1\right\}, \neg, \wedge, \rightarrow,\left\{\frac{1}{2}, 1\right\}\right),
$$

com o conjunto de valores designados $D=\left\{\frac{1}{2}, 1\right\}$ e com a seguinte relação de consequência semântica.

Seja $\operatorname{Var}(L P T)=\left\{p_{1}, p_{2}, p_{3}, \ldots\right\}$ o conjunto das variáveis proposicionais de LPT. Uma valoração para LPT é qualquer função:

$$
v: \operatorname{Var}(L P T) \rightarrow\left\{0, \frac{1}{2}, 1\right\},
$$

a qual é estendida de modo único para o conjunto For $(L P T)$ segundo os operadores introduzidos acima.

Se $\Gamma \subseteq$ For $(L P T)$, então $v(\Gamma)=\{v(\gamma): \gamma \in \Gamma\}$ e a implicação lógica ou consequência semântica de LPT é definida como segue.

Para $\Gamma \cup\{\varphi\} \subseteq$ For $(L P T)$, o conjunto $\Gamma$ implica $\varphi$ quando para toda LPT-valoração $v$, se $v(\Gamma) \subseteq D$, então $v(\varphi) \in D$, isto é:

$$
\Gamma \vDash \varphi \Longleftrightarrow v(\Gamma) \subseteq D \Rightarrow v(\varphi) \in D
$$

Decorre desta definição de valoração que toda fórmula de LPT válida segundo uma valoração $v: \operatorname{Var}(L P T) \rightarrow\left\{0, \frac{1}{2}, 1\right\}$ é também válida segundo a restrição booleana de $v$, isto é, segundo $v: \operatorname{Var}(L P T) \rightarrow\{0,1\}$ com os significados booleanos dos operadores $\neg, \wedge \mathrm{e} \rightarrow$, em que é apagado o valor $\frac{1}{2}$. Assim, toda fórmula LPT-válida é uma tautologia.

Podemos construir tabelas de verdade de fórmulas de LPT, que por ser uma lógica trivalente, tem como número de linhas algum múltiplos de 3 . Vejamos alguns exemplos como em (Feitosa e Silvestrini, 2016): 
(a) $\varphi \rightarrow(\psi \rightarrow \varphi)$ :

\begin{tabular}{|c|c|c|c|c|}
\hline$\varphi$ & $\rightarrow$ & $(\psi$ & $\rightarrow$ & $\varphi)$ \\
\hline 0 & $\mathbf{1}$ & 0 & 1 & 0 \\
\hline 0 & $\mathbf{1}$ & $\frac{1}{2}$ & 0 & 0 \\
\hline 0 & $\mathbf{1}$ & 1 & 0 & 0 \\
\hline$\frac{1}{2}$ & $\mathbf{1}$ & 0 & 1 & $\frac{1}{2}$ \\
\hline$\frac{1}{2}$ & $\mathbf{1}$ & $\frac{1}{2}$ & 1 & $\frac{1}{2}$ \\
\hline$\frac{1}{2}$ & $\mathbf{1}$ & 1 & 1 & $\frac{1}{2}$ \\
\hline 1 & $\mathbf{1}$ & 0 & 1 & 1 \\
\hline 1 & $\mathbf{1}$ & $\frac{1}{2}$ & 1 & 1 \\
\hline 1 & $\mathbf{1}$ & 1 & 1 & 1 \\
\hline
\end{tabular}

(b) $\varphi \vee(\varphi \rightarrow \psi)$ :

\begin{tabular}{|c|c|c|c|c|}
\hline$\varphi$ & $\vee$ & $(\varphi$ & $\rightarrow$ & $\psi)$ \\
\hline 0 & $\mathbf{1}$ & 0 & 1 & 0 \\
\hline 0 & $\mathbf{1}$ & 0 & 1 & $\frac{1}{2}$ \\
\hline 0 & $\mathbf{1}$ & 0 & 1 & 1 \\
\hline$\frac{1}{2}$ & $\frac{1}{\mathbf{2}}$ & $\frac{1}{2}$ & 0 & 0 \\
\hline$\frac{1}{2}$ & $\mathbf{1}$ & $\frac{1}{2}$ & 1 & $\frac{1}{2}$ \\
\hline$\frac{1}{2}$ & $\mathbf{1}$ & $\frac{1}{2}$ & 1 & 1 \\
\hline 1 & $\mathbf{1}$ & 1 & 0 & 0 \\
\hline 1 & $\mathbf{1}$ & 1 & 1 & $\frac{1}{2}$ \\
\hline 1 & $\mathbf{1}$ & 1 & 1 & 1 \\
\hline
\end{tabular}

(c) $\varphi \vee \neg \varphi$ :

\begin{tabular}{|c|c|c|}
\hline$\varphi$ & $\vee$ & $\neg \varphi$ \\
\hline 0 & $\mathbf{1}$ & 1 \\
\hline$\frac{1}{2}$ & $\frac{1}{\mathbf{2}}$ & $\frac{1}{2}$ \\
\hline 1 & $\mathbf{1}$ & 0 \\
\hline
\end{tabular}

(d) Cada fórmula $\sigma$ do tipo $\varphi \wedge \neg \varphi \wedge \circ \varphi$ é contraditória:

\begin{tabular}{|c|c|c|c|}
\hline$\varphi$ & $\neg \varphi$ & $\circ \varphi$ & $\sigma$ \\
\hline 0 & 1 & 1 & 0 \\
\hline$\frac{1}{2}$ & $\frac{1}{2}$ & 0 & 0 \\
\hline 1 & 0 & 1 & 0 \\
\hline
\end{tabular}

Contudo, algumas fórmulas tautológicas bem conhecidas não são LPT-válidas. Vejamos algumas delas: 
(e) $(\varphi \wedge \neg \varphi) \rightarrow \psi$.

Tomemos uma valoração $v$ tal que $v(\varphi)=\frac{1}{2}$ e $v(\psi)=0$. Daí, $v((\varphi \wedge \neg \varphi) \rightarrow \psi)=\left(\frac{1}{2} \wedge \frac{1}{2}\right) \rightarrow$ $0=\frac{1}{2} \rightarrow 0=0$.

(f) $\neg \varphi \rightarrow(\varphi \rightarrow \psi)$.

Tomemos, mais uma vez, uma valoração $v$ tal que $v(\varphi)=\frac{1}{2}$ e $v(\psi)=0$. Daí, $v(\neg \varphi \rightarrow(\varphi \rightarrow$ $\psi))\left(\frac{1}{2} \rightarrow\left(\frac{1}{2} \rightarrow 0\right)\right)=\frac{1}{2} \rightarrow 0=0$.

(g) $(\varphi \rightarrow \psi) \rightarrow(\neg \psi \rightarrow \neg \varphi)$.

Tomemos uma valoração $v$ tal que $v(\varphi)=1$ e $v(\psi)=\frac{1}{2}$. Daí, $v((\varphi \rightarrow \psi) \rightarrow(\neg \psi \rightarrow \neg \varphi))=$ $\left(1 \rightarrow \frac{1}{2}\right) \rightarrow\left(\frac{1}{2} \rightarrow 0\right)=1 \rightarrow 0=0$.

Proposição 2.1 Se v : For $(L P T) \rightarrow\left\{0, \frac{1}{2}, 1\right\}$ é uma LPT-valoração, então:

(i) $v(\varphi) \in D \Leftrightarrow v(\varphi)=\frac{1}{2}$ ou $v(\varphi)=1$;

(ii) $v(\neg \varphi) \in D \Leftrightarrow v(\varphi)=\frac{1}{2}$ ou $v(\varphi)=0$;

(iii) $v(\circ \varphi) \in D \Leftrightarrow v(\varphi)=0$ ou $v(\varphi)=1$.

Demonstração: Imediata das tabelas dos operadores de LPT.

A lógica LPT de acordo com (Feitosa e Silvestrini, 2016) é dada pelo seguinte sistema axiomático:

Esquemas de Axiomas:

(A1) $\varphi \rightarrow(\psi \rightarrow \varphi)$

(A2) $(\varphi \rightarrow(\psi \rightarrow \sigma)) \rightarrow((\varphi \rightarrow \psi) \rightarrow(\varphi \rightarrow \sigma))$

(A3) $(\sigma \rightarrow \varphi) \rightarrow((\sigma \rightarrow \psi) \rightarrow(\sigma \rightarrow(\varphi \wedge \psi)))$

(A4) $(\varphi \wedge \psi) \rightarrow \varphi$

(A5) $(\varphi \wedge \psi) \rightarrow \psi$

(A6) $\varphi \rightarrow(\varphi \vee \psi)$

(A7) $\psi \rightarrow(\varphi \vee \psi)$

(A8) $(\varphi \rightarrow \sigma) \rightarrow((\psi \rightarrow \sigma) \rightarrow((\varphi \vee \psi) \rightarrow \sigma)))$

(A9) $\varphi \vee(\varphi \rightarrow \psi)$

(A10) $\varphi \vee \neg \varphi$

(A11) $\neg \neg \varphi \rightarrow \varphi$

$(\mathrm{A} 12) \circ \varphi \rightarrow(\varphi \rightarrow(\neg \varphi \rightarrow \psi))$

$(\mathrm{A} 13) \neg \circ \varphi \rightarrow(\varphi \wedge \neg \varphi)$

$(\mathrm{A} 14) \circ(\varphi \rightarrow \psi)$

$(\mathrm{A} 15)(\circ \varphi \wedge \circ \psi) \rightarrow \circ(\varphi \wedge \psi)$

(A16) $\circ \varphi \rightarrow \circ \neg \varphi$.

Regra de Dedução:

(MP) $\varphi, \varphi \rightarrow \psi \vdash \psi$. 
Feitosa e Silvestrini (2016) demonstram que o sistema dedutivo, acima, da lógica LPT é correto e completo segundo a semântica matricial $\mathscr{M}_{L P T}$.

Portanto, temos o seguinte teorema.

Teorema 2.2 $\Gamma \vdash \varphi \Leftrightarrow \Gamma \vDash \varphi$.

\section{O método de tableaux analíticos}

Os sistemas de tableaux para a Lógica Clássica, proposicional e quantificacional, foram muito bem investigados e apresentados por Smullyan (1968).

A origem de tal método, entretanto, está baseada nos trabalhos de Gentzen (1935), quando da introdução dos sistemas de provas conhecidos como cálculos de sequentes. Tais sistemas de prova seguem o princípio da subfórmula, no qual estabelece que se uma fórmula tem uma demonstração, então ela possui uma demonstração em que ocorrem apenas subfórmulas da fórmula inicial.

Dada esta característica, Smullyan (1968) denominou seus tableaux de analíticos.

O trabalho de Gentzen foi desenvolvido posteriormente por E. W. Beth (1959), que introduziu o método de tableaux semânticos, os quais também fazem uso do princípio da subfórmula.

Smullyan (1968) ao introduzir o sistema de tableaux analíticos, buscou estabelecer as relações desse com os métodos originais de Gentzen.

Além desses autores, J. Hintikka (1955) também inspirou Smullyan(1968), de tal modo que o método dos tableaux analíticos de Smullyan pode ser considerado uma variante dos métodos de prova de Hintikka.

Uma característica principal deste sistema de prova é que se trata de um método de refutação, ou seja, para demonstrarmos que uma fórmula $\varphi$ é válida, tomamos como primeiro passo supor que ela não é válida.

A partir daí aplicamos as regras de expansão do tableau que, a cada passo, gera como consequências apenas subfórmulas da fórmula considerada. Como cada fórmula é finita, então este procedimento depois de um número finito de passos tem que estar exaurido. Nesse ponto podemos fazer a análise da validade ou não da fórmula inicial.

Iniciamos considerando que $\varphi$ não é válida. $\mathrm{Na}$ análise quando o procedimento está concluído, se encontramos alguma contradição em cada ramo do tableau, então concluímos que não há um caminho ou um ramo que corrobore a suposição inicial de não validade de $\varphi$. Logo, a conclusão é que $\varphi$ é verdadeira.

Caso tal fato não ocorra, ou seja, o tableau apresenta algum ramo aberto, isto é, sem alguma contradição na expansão da negação de $\varphi$, então este mesmo ramo serve como um contraexemplo de valoração para fórmula $\varphi$, que a faz falsa.

Ademais, o método de tableaux analíticos é caracterizado como um algoritmo e, assim, é um sistema de decisão para fórmulas válidas de uma determinada lógica, do mesmo modo que as tabelas de verdade são para a lógica proposicional clássica. Com a enorme vantagem de ser um procedimento muito mais breve e econômico.

A base de todo sistema de tableaux analíticos está nas regras de expansão ou regras para a construção dos tableaux, as quais permitem a análise das fórmulas de uma linguagem $\mathbf{L}$. 
A noção de expansão é justamente agir sobre uma fórmula em questão de modo a considerar as suas subfórmulas na geração de um ramo do tableau, como numa árvore.

Empregamos a palavra 'ramo' para designar um caminho ou uma possibilidade de análise das subfórmulas da fórmula considerada.

Smullyan (1968) apresenta seu desenvolvimento como sendo uma árvore ordenada e diádica, donde surge naturalmente o termo 'ramo'.

Ele apresentou seu método de tableaux tanto para a lógica proposicional clássica (LPC), como para a lógica de primeira ordem (FOL).

Mostramos, agora, apenas os tableaux para uma lógica proposicional clássica. Mais detalhes podem ser encontrados em Smullyan (1968) e em Silva, Finger e Melo (2006). Usaremos as regras do segundo texto, com fórmulas marcadas, em que $\mathrm{T}$ representa a verdade (truth) e $\mathrm{F}$ representa o falso. A linguagem $\mathbf{L}$ conta com os seguintes operadores lógicos $\mathbf{L}=(\neg, \wedge, \vee, \rightarrow)$ :

As fórmulas neste sistema de prova podem ser classificadas da seguinte forma:

Fórmulas do tipo $A$ : as consequências das fórmulas do tipo $A$ são consequências diretas, isto é, permanecem no mesmo ramo e não geram bifurcações. As regras de expansão que contém esta categoria de fórmulas são denominadas regras do tipo conjuntivo.

Fórmulas do tipo $B$ : neste caso, as fórmulas do tipo $B$ não são diretas e, assim, elas bifurcam em dois ramos distintos, sendo que cada um deles é uma possibilidade de análise da fórmula dada. As regras de expansão que têm fórmulas do tipo $\mu$ são chamadas de regras do tipo disjuntivo.

Apresentamos, a seguir, as regras de expansão do tableaux analíticos para a LPC.

\section{Regras do tipo conjuntivo:}

\begin{tabular}{|c|c|c|c|c|c|c|c|c|c|}
\hline & & & & $\mathrm{T}$ & $\alpha \wedge \beta$ & $\mathrm{F}$ & $\alpha \vee \beta$ & $\mathrm{F}$ & $\alpha \rightarrow \beta$ \\
\hline $\mathrm{F}$ & $\alpha$ & & $\alpha$ & $\mathrm{T}$ & $\alpha$ & $\mathrm{F}$ & $\alpha$ & $\mathrm{T}$ & $\alpha$ \\
\hline
\end{tabular}

\section{Regras do tipo disjuntivo:}

$$
\frac{\mathrm{F} \alpha \wedge \beta}{\mathrm{F} \alpha \mid \mathrm{F} \beta} \quad \frac{\mathrm{T} \alpha \vee \beta}{\mathrm{T} \alpha \mid \mathrm{T} \beta} \quad \frac{\mathrm{T} \alpha \rightarrow \beta}{\mathrm{F} \alpha \mid \mathrm{T} \beta} .
$$

Após a aplicação de todas as regras de expansão possíveis num tableau, podemos encontrar uma contradição num ramo, quando para alguma fórmula $\varphi$, ocorrem no ramo as fórmulas marcadas T $\varphi$ e F $\varphi$. Neste caso, dizemos que o ramo é fechado. Do contrário, dizemos que o ramo é aberto.

Se todos os ramos do tableau são fechados, então temos um tableau fechado e, deste modo, concluímos que a fórmula inicial é válida. Do contrário, a fórmula inicial não é válida.

Muitos exemplos podem ser encontrados nos dois textos mencionados. Os tableaux, da próxima seção, também servirão de exemplo e, para o caso clássico, basta considerarmos $\mathrm{T}=$ 1 e $\mathrm{F}=0$. 


\section{Tableaux para a LPT}

Agora introduzimos o sistema de tableaux analíticos para a lógica da verdade pragmática (LPT), que originalmente foi apresentada por Silvestrini (2011) em um sistema axiomático.

Denotamos o nosso sistema de tableaux para LPT por TPT.

As regras de expansão para o sistema TPT podem ser classificadas em três tipos, os quais seguem abaixo.

Fórmulas do tipo $\alpha$ : são fórmulas em que temos consequências diretas e, com isso, as suas consequências não se ramificam, mas permanecem no mesmo ramo.

Fórmulas do tipo $\beta$ : neste caso, as consequências se ramificam em dois ramos distintos.

Fórmulas do tipo $\gamma$ : são fórmulas em que as consequências se ramificam em três ramos distintos.

Regras do tipo $\gamma$ são complemente novas para os tableuax clássicos, os quais são diádicos e, portanto, se dividem no máximo em dois ramos, para cada regra.

Definição 4.1 Um ramo de tableau em TPT é fechado quando ocorrer um dos seguintes casos:

(i) uma fórmula ocorre com valores distintos no ramo;

(ii) se ocorre no ramo a fórmula marcada $\frac{1}{2} \circ \varphi$;

(iii) se ocorre no ramo a fórmula marcada $\frac{1}{2} \quad \varphi \rightarrow \psi$.

Como indicam as tabelas de LPT, não há casos em que qualquer fórmula de consistência o ou de condicional $\rightarrow$ assuma o valor $\frac{1}{2}$. Contudo, num tableau pode ser que nalguma expansão surja uma tal situação não sustentável em LPT. Por isso a inclusão das condições (ii) e (iii) na definição do fechamento de um ramo em TPT.

Definição 4.2 Um tableau do sistema TPT é fechado se todos os seus ramos são fechados.

Introduzimos as regras de expansão para o nosso sistema TPT.

\section{Negação:}

$$
\begin{aligned}
& {[0 \neg] \frac{0 \quad \neg \varphi}{1 \quad \varphi}} \\
& {\left[\frac{1}{2} \neg\right] \frac{\frac{1}{2} \quad \neg \varphi}{\frac{1}{2} \quad \varphi}} \\
& {[1 \neg] \frac{1}{1} \frac{\neg \varphi}{0}}
\end{aligned}
$$

\section{Consistência:}

$$
[0 \circ] \frac{0}{\frac{1}{2} \varphi} \frac{\varphi}{\frac{1}{2}} \quad[1 \quad \circ] \frac{1 \circ \varphi}{0 \varphi \mid 1 \varphi}
$$




\section{Conjunção:}

$$
[0 \wedge] \frac{0 \varphi \wedge \psi}{0 \varphi \mid 0 \psi} \quad\left[\frac{1}{2} \wedge\right] \begin{gathered}
\frac{1}{2} \varphi \wedge \psi \\
{{2} \varphi\left|\frac{1}{2} \varphi\right|} \mid 1 \varphi } \\
1 \psi\left|\frac{1}{2} \psi\right| \frac{1}{2} \psi
\end{gathered}
$$

\section{Disjunção:}

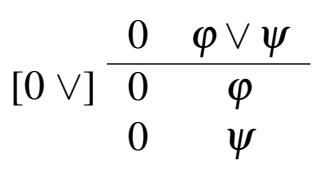

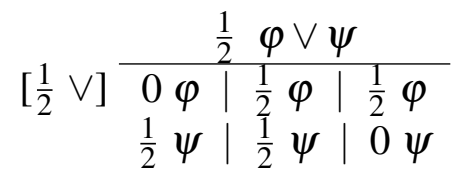

$[1 \vee] \frac{1 \varphi \vee \psi}{1 \varphi}$

\section{Condicional:}

$$
[0 \rightarrow] \frac{0 \varphi \rightarrow \psi}{0 \psi} \quad[1 \rightarrow] \frac{1 \varphi \rightarrow \psi}{0 \varphi\left|\frac{1}{2} \psi\right| 1 \psi}
$$

As regras de expansão no sistema TPT foram obtidas por meio da análise das matrizes trivalentes da LPT, introduzidas por Silvetsrini (2011).

\section{Adequação de TPT}

Precisamos verificar que o nosso sistema de tableaux é equivalente ao sistema dedutivo LPT, isto é, que TPT não deduz mais e nem menos que LPT.

Assim, temos que comprovar que todas as deduções que obtemos em TPT também são obtidas em na LPT e vice-versa e, portanto, teremos a seguinte equivalência:

$$
\Gamma \Vdash \varphi \Leftrightarrow \Gamma \vdash \varphi \Leftrightarrow \Gamma \vDash \varphi .
$$

Como a segunda equivalência $\Gamma \vdash \varphi \Leftrightarrow \Gamma \vDash \varphi$ está em (Feitosa, Silvestrini, 2016), então seguiremos o seguinte caminho:

$$
\begin{gathered}
\Gamma \vdash \varphi \Leftrightarrow \Gamma \models \varphi \\
\Downarrow \quad \Uparrow \\
\Gamma \Vdash \varphi
\end{gathered}
$$

Teorema 5.1 Se $\Gamma \vdash \varphi \Rightarrow \Gamma \Vdash \varphi$

Demonstração: Demonstração por indução no comprimento de dedução $\Gamma \vdash \varphi$.

Se $n=1$, então temos os seguintes casos: $\varphi \in \Gamma$ ou $\varphi$ é um axioma.

Se $\varphi \in \Gamma$, temos $\Gamma \Vdash \varphi$, pois $\varphi$ ocorre com valores distintos no tableau e por isso o tableau fecha.

Se $\varphi$ é um axioma, então $\Vdash \varphi$ e, portanto, $\Gamma \Vdash \varphi$. Diante disso, provamos agora que cada axioma da LPT gera um tableau fechado em TPT. 
(A1) $\varphi \rightarrow(\psi \rightarrow \varphi)$

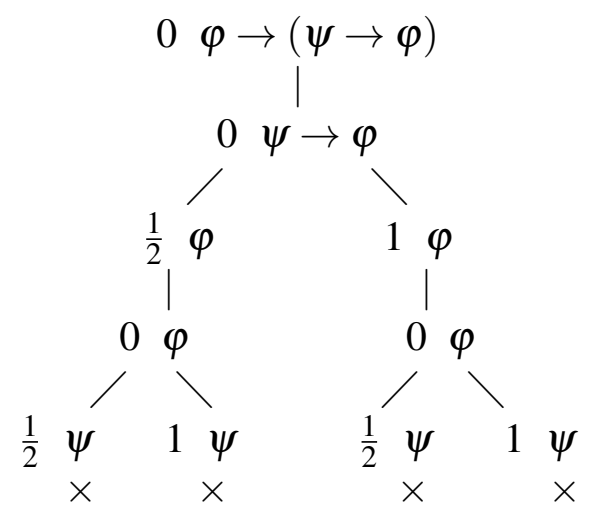

(A4) $(\varphi \wedge \psi) \rightarrow \varphi$

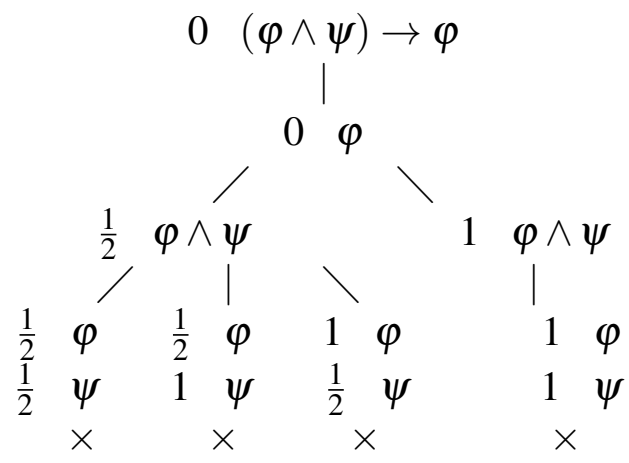

(A5) $(\varphi \wedge \psi) \rightarrow \psi$

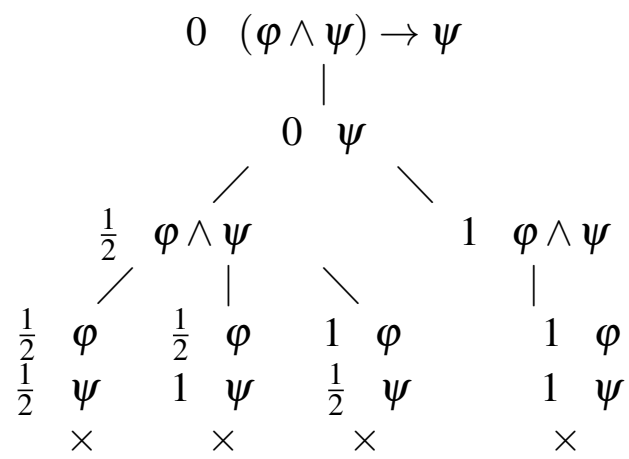

(A2) $(\varphi \rightarrow(\psi \rightarrow \sigma)) \rightarrow((\varphi \rightarrow \psi) \rightarrow(\varphi \rightarrow \sigma))$

$$
\begin{gathered}
0(\varphi \rightarrow(\psi \rightarrow \sigma)) \rightarrow((\varphi \rightarrow \psi) \rightarrow(\varphi \rightarrow \sigma)) \\
0 \quad(\varphi \rightarrow \psi) \rightarrow(\varphi \rightarrow \sigma) \\
\frac{1}{2} \varphi \rightarrow(\psi \rightarrow \sigma) \quad 1 \varphi \rightarrow(\psi \rightarrow \sigma)
\end{gathered}
$$




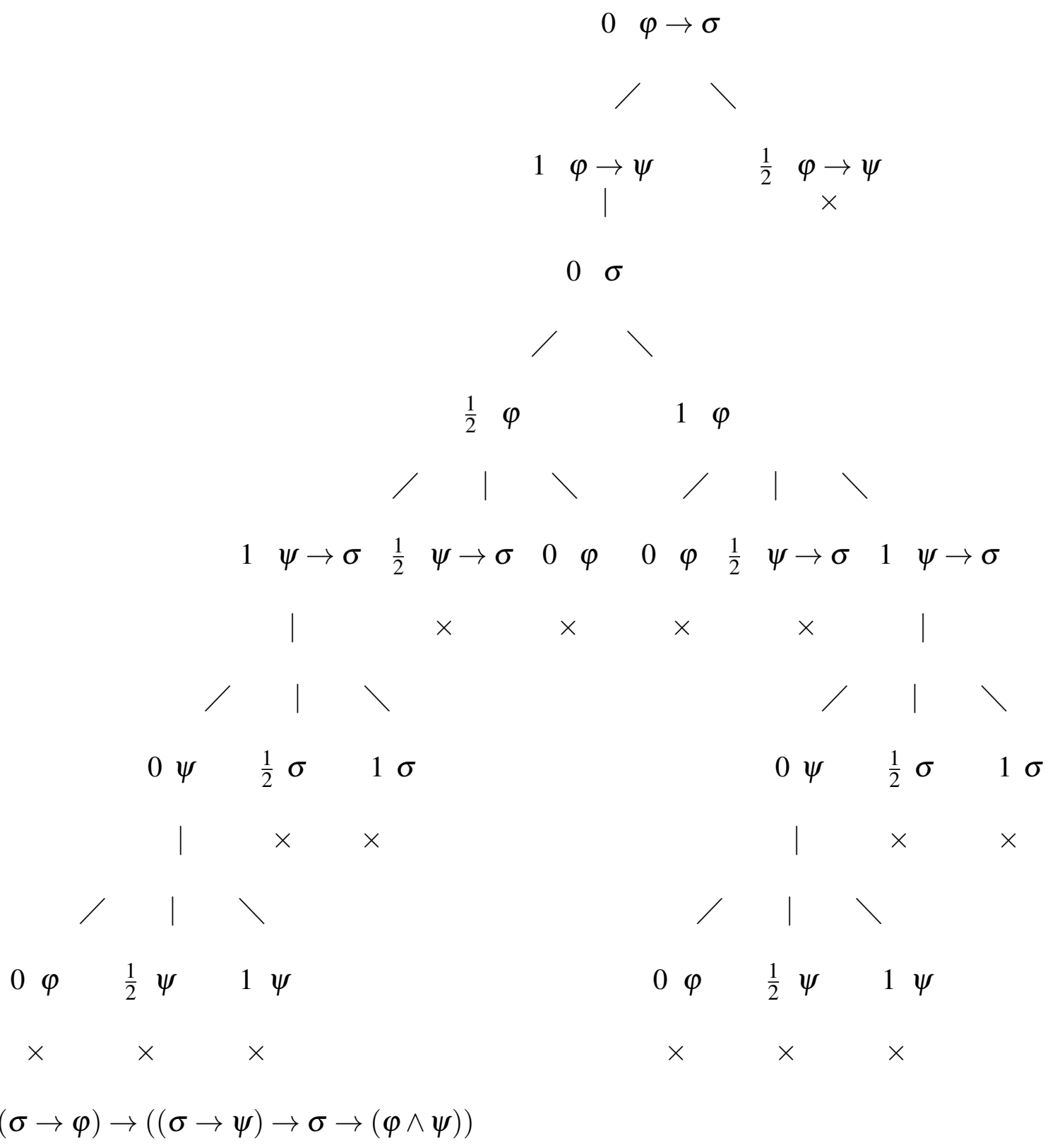

Feitosa e Silvestrini (2016) demonstraram que o axioma (A3) é equivalente a seguinte fórmula $\varphi \rightarrow(\psi \rightarrow(\varphi \wedge \psi))$. Por questão de economia no tamanho, faremos o tableau para esta fórmula. Certamente, verificamos também a validade de (A3). 


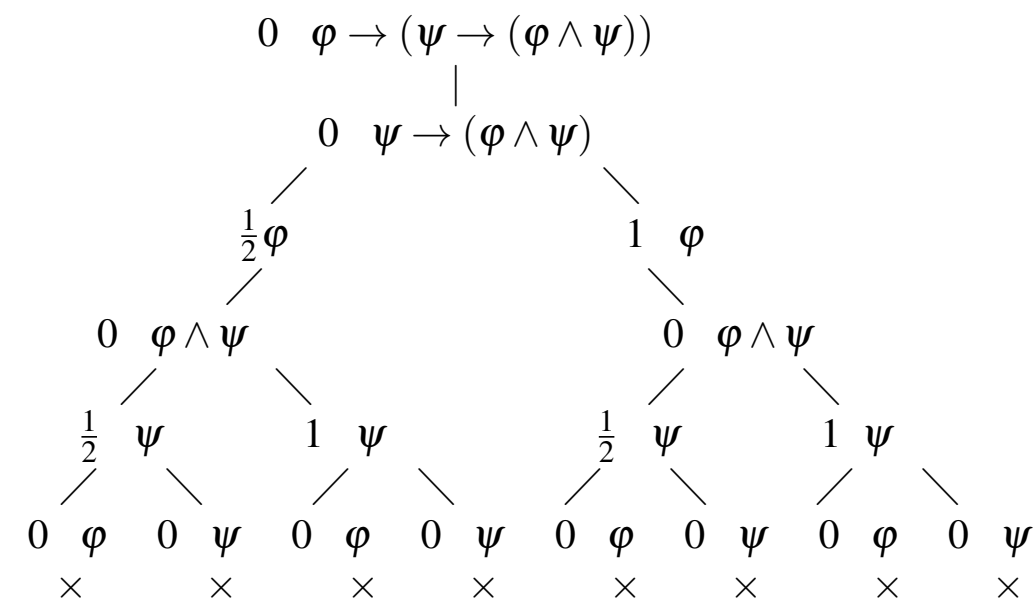

(A6) $\varphi \rightarrow(\varphi \vee \psi)$

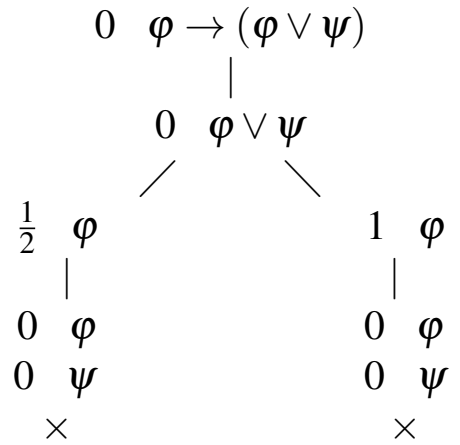

(A7) $\psi \rightarrow(\varphi \vee \psi)$

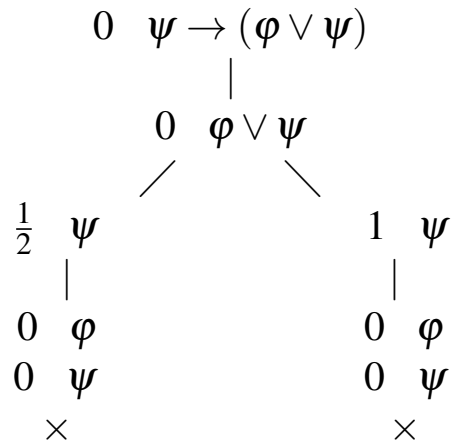

(A9) $\varphi \vee(\varphi \rightarrow \psi)$ 


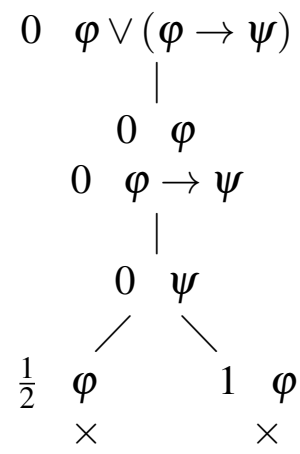

$(\mathrm{A} 14) \circ(\varphi \rightarrow \psi)$

$$
\begin{gathered}
0 \circ(\varphi \rightarrow \psi) \\
\frac{1}{2} \quad \varphi \rightarrow \psi
\end{gathered}
$$

(A8) $(\varphi \rightarrow \sigma) \rightarrow((\psi \rightarrow \sigma) \rightarrow((\varphi \vee \psi) \rightarrow \sigma)$

$$
\begin{aligned}
& 0 \quad(\varphi \rightarrow \sigma) \rightarrow((\psi \rightarrow \sigma) \rightarrow(\varphi \vee \psi \rightarrow \sigma)) \\
& 0 \quad((\psi \rightarrow \sigma) \rightarrow(\varphi \vee \psi \rightarrow \sigma)) \\
& \frac{1}{2} \varphi \rightarrow \sigma \\
& 1 \varphi \rightarrow \sigma
\end{aligned}
$$

$\times$

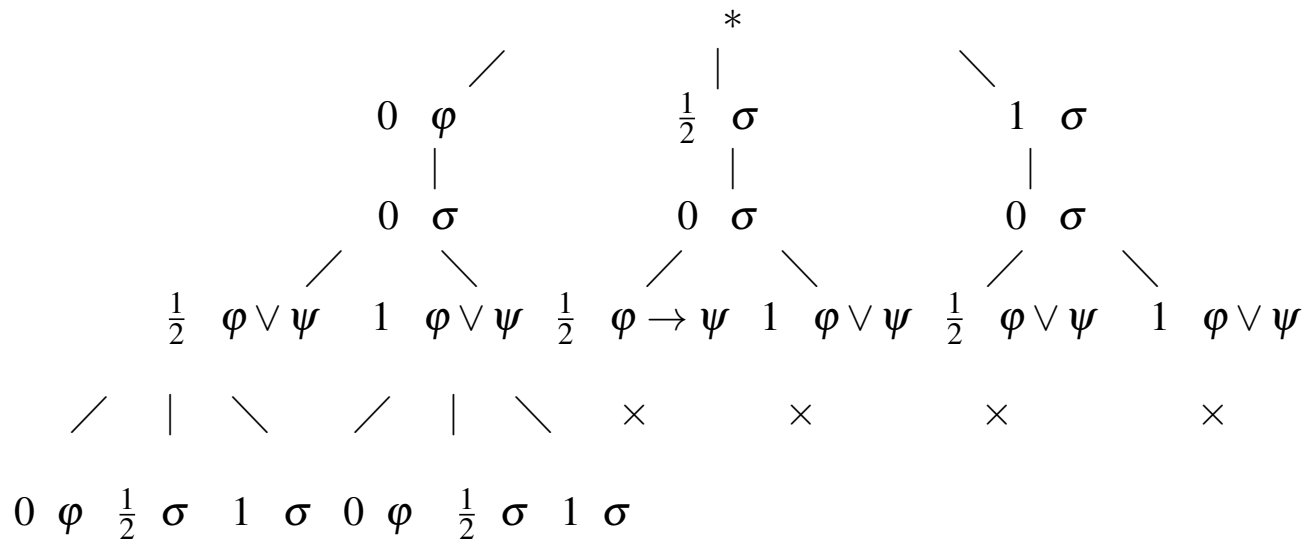


$* * \times \times * * \times \times$

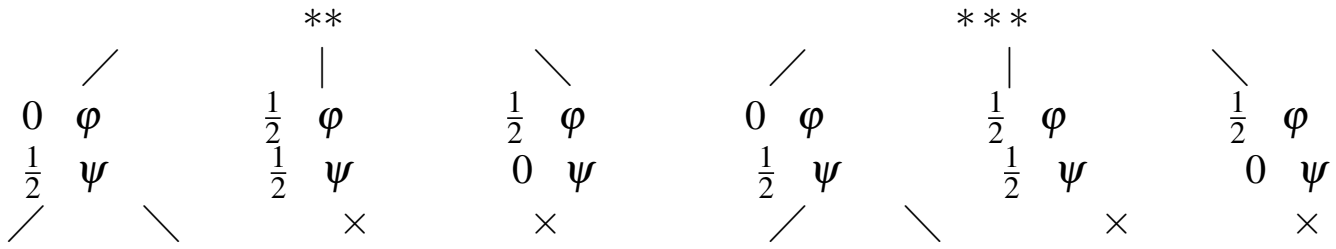
$0 \quad \frac{1}{2}, 1 \quad \sigma$
$0 \quad \psi \quad \frac{1}{2}, 1 \quad \sigma$
$\times \quad \times, \times$
$\times$
$\times, \times$

(A10) $\varphi \vee \neg \varphi$

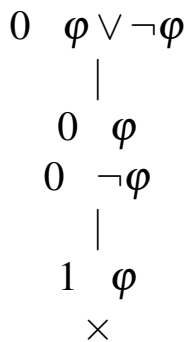

$(\mathrm{A} 12) \circ \varphi \rightarrow(\varphi \rightarrow(\neg \varphi \rightarrow \psi))$

$0 \circ \varphi \rightarrow(\varphi \rightarrow(\neg \varphi \rightarrow \psi))$

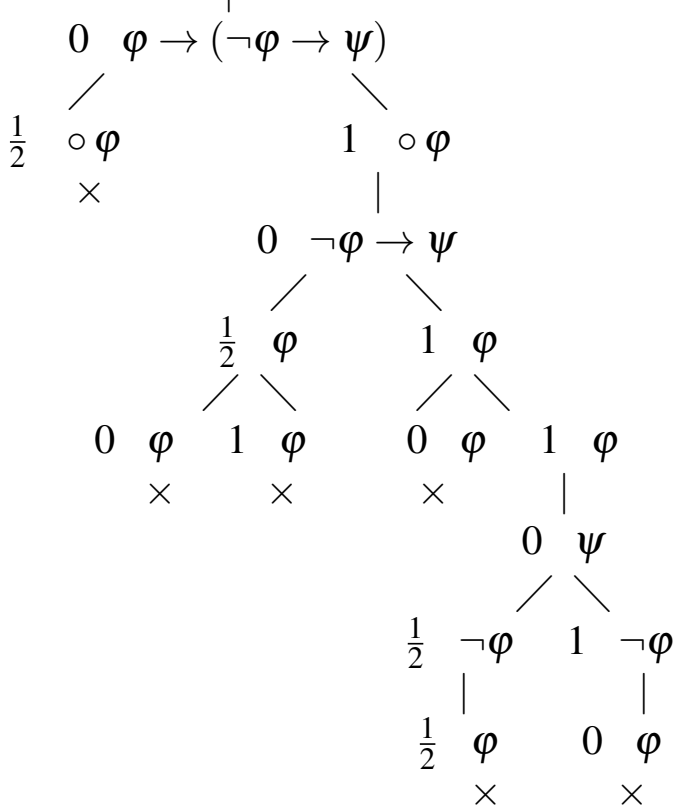


$(\mathrm{A} 13) \neg \circ \varphi \rightarrow(\varphi \wedge \neg \varphi)$

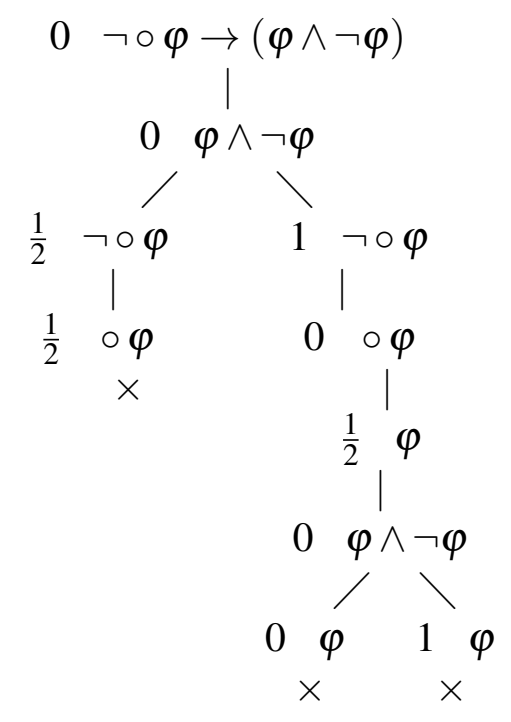

(A11) $\neg \neg \varphi \rightarrow \varphi$

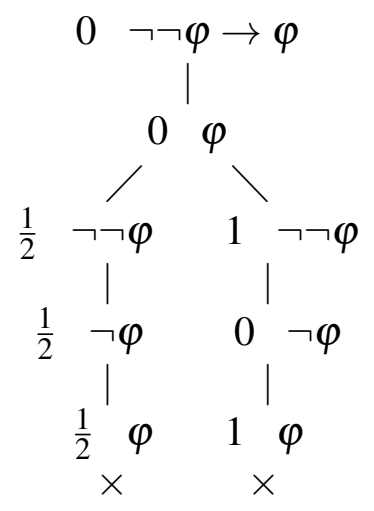

$(\mathrm{A} 15)(\circ \varphi \wedge \circ \psi) \rightarrow \circ(\varphi \wedge \psi)$

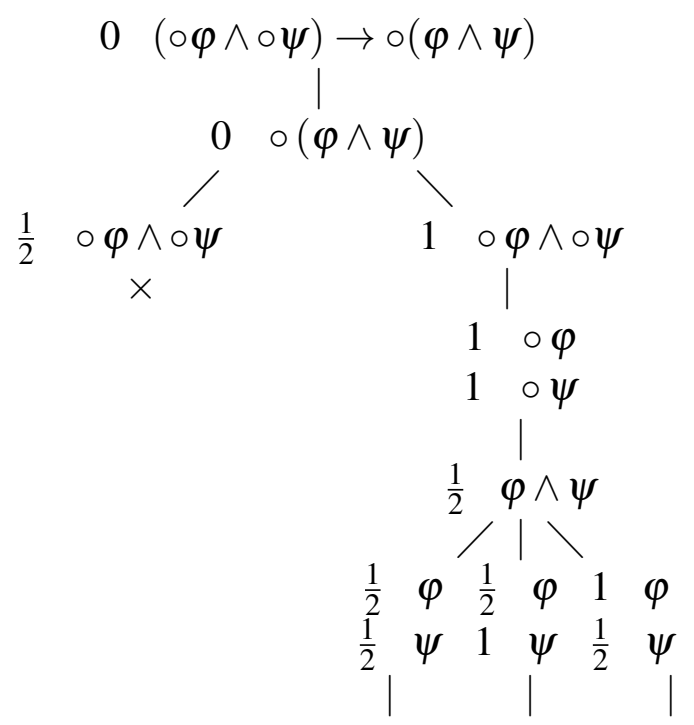




$$
\begin{array}{cccccc}
0,1 & \varphi & 0,1 & \varphi & 0,1 & \psi \\
\times & & \times & \times
\end{array}
$$

Nesta último passo, a notação $0,1 \quad \varphi$ indica que vale $0 \quad \varphi$ oи $1 \varphi$.

$(\mathrm{A} 16) \circ \varphi \rightarrow \circ \neg \varphi$

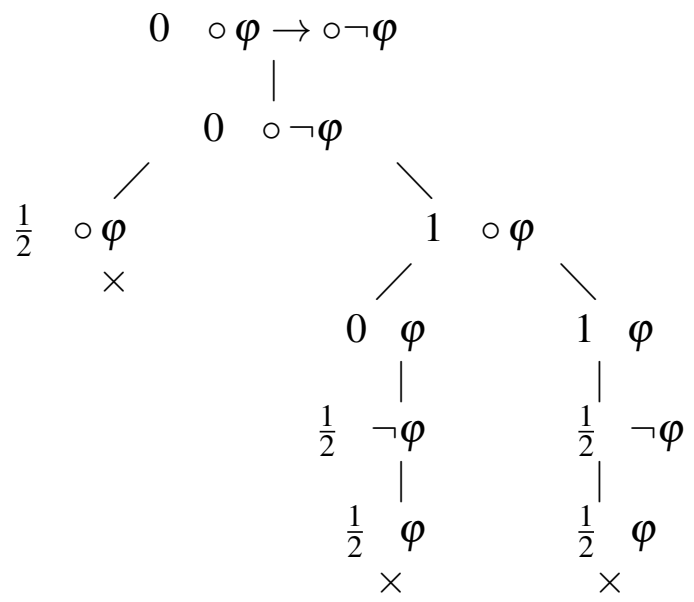

Se $n>1$, então no último passo da dedução aplicamos a regra MP. Assim, temos $\Gamma \vdash \sigma \rightarrow \varphi$ $e \Gamma \vdash \sigma$ donde concluímos que $\Gamma \vdash \varphi$.

Por hipótese de indução, temos que $\Gamma \Vdash \sigma \rightarrow \varphi$ e $\Gamma \Vdash \sigma$. Daí, segue que para toda valoração $v$, se $v(\Gamma) \subseteq\left\{\frac{1}{2}, 1\right\}$ então $v(\sigma \rightarrow \varphi) \neq 0$ e $v(\sigma) \neq 0$.

Deste modo, consideraremos o tableau $\Gamma, \sigma, \sigma \rightarrow \varphi \Vdash \varphi$. Temos quatro condições possíveis para as premissas válidas $\sigma e \sigma \rightarrow \varphi$ :
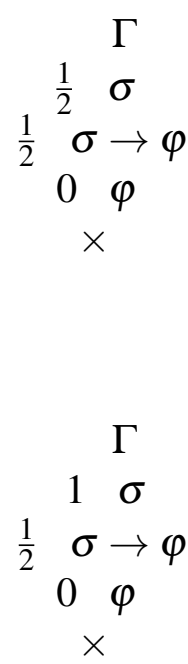
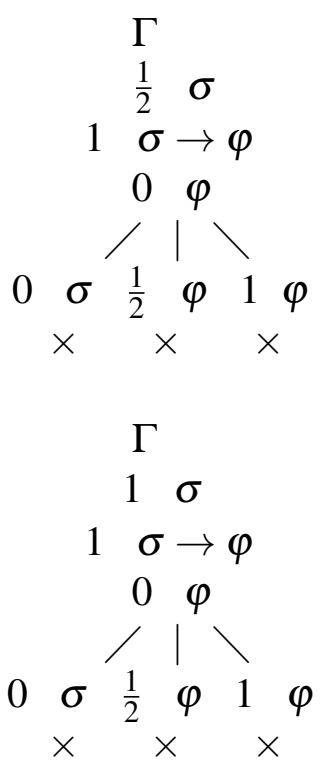

Portanto, $\Gamma \Vdash \varphi$. 


\footnotetext{
Para obtermos uma equivalência entre os dois sistemas, ainda temos que provar: $\Gamma \Vdash \varphi \Rightarrow$ $\Gamma \vDash \varphi$.
}

Contudo, antes disso, precisaremos de algumas definições e alguns resultados.

Definição 5.2 Um conjunto $\Theta$ de fórmulas sinalizadas é saturado para baixo se satisfaz as seguintes condições:

(a) nenhuma fórmula sinalizada ocorre em $\Theta$ com dois valores distintos;

(b) se em $\Theta$ ocorre alguma fórmula sinalizada do tipo $\alpha$, então $\alpha_{1} \in \Theta$ e $\alpha_{2} \in \Theta$;

(c) se em $\Theta$ ocorre alguma fórmula sinalizada do tipo $\beta$, então $\beta_{1} \in \Theta$ ou $\beta_{2} \in \Theta$;

(d) se em $\Theta$ ocorre alguma fórmula sinalizada do tipo $\gamma$, então $\gamma_{1} \in \Theta$ ou $\gamma_{2} \in \Theta$ ou $\gamma_{3} \in \Theta$.

Lema 5.3 Todo ramo saturado e aberto de um tableau é um conjunto saturado para baixo.

Demonstração: Como o ramo é aberto, então nenhuma fórmula aparece no ramo com duas valorações distintas, o que satisfaz a condição (a) da definição de conjunto saturado para baixo.

Além disso, como o ramo é saturado, segue que todas as possiveis regras do tableau já foram utilizadas e o tableau não pode mais ser expandido.

Logo, se existe uma fórmula do tipo $\alpha$ no ramo, então $\alpha_{1}$ e $\alpha_{2}$ também estão no ramo, o que atende a condição $(b)$.

Pelo mesmo motivo, se há uma fórmula do tipo $\beta$ no ramo, então ou $\beta_{1}$ ou $\beta_{2}$ está no ramo, o que cumpre a condição (c).

De modo análogo, também pela saturação, se ocorre no ramo uma fórmula do tipo $\gamma$, segue que ou $\gamma_{1}$ ou $\gamma_{2}$ ou $\gamma_{3}$ está no ramo, o que contempla o última condição $(d)$.

Agora, estenderemos a noção de valoração para as fórmulas sinalizadas.

Definição 5.4 Se v é uma valoração e $k \in\left\{0, \frac{1}{2}, 1\right\}$, então a fórmula sinalizada $k \varphi$ é distinguida segundo a valoração $v$, o que é denotado por $k \varphi \in D$, se $v(\varphi)=k$.

Assim, $k \varphi \in D \Leftrightarrow v(\varphi)=k$.

Definição 5.5 Uma valoração $v$ satisfaz um conjunto $\Theta$ de fórmulas sinalizadas se para toda fórmula sinalizada $k \psi$ que ocorre em $\Theta$, tem-se $k \psi \in D$.

Definição 5.6 Um conjunto $\Theta$ de fórmulas sinalizadas é satisfatível se existe uma valoração $v$ tal que $v(\Theta) \subseteq D$, ou seja, para toda $\psi \in \Theta, k \psi \in D$.

Lema 5.7 Se $\Theta$ é um conjunto satisfatível de fórmulas sinalizadas, então:

(i) se uma fórmula do tipo $\alpha$ está em $\Theta$, então $\Theta \cup\left\{\alpha_{1}, \alpha_{2}\right\}$ é satisfatível;

(ii) se uma fórmula do tipo $\beta$ está em $\Theta$, então $\Theta \cup\left\{\beta_{1}\right\}$ é satisfatível ou $\Theta \cup\left\{\beta_{2}\right\}$ é satisfativel;

(iii) se uma fórmula do tipo $\gamma$ está em $\Theta$, então ou $\Theta \cup\left\{\gamma_{1}\right\}$ é satisfativel, ou $\Theta \cup\left\{\gamma_{2}\right\}$ é satisfativel, ou $\Theta \cup\left\{\gamma_{3}\right\}$ é satisfativel.

Demonstração: (i) Tomemos a fórmula de consistência do tipo $\alpha$, isto é, $0 \circ \varphi$. Como o conjunto $\Theta$ é satisfatível, então existe uma valoração v tal que $v(\Theta) \subseteq D$. Daí, $v(\circ \varphi)=0$ e, então $v(\varphi)=\frac{1}{2}$ $e$, portanto, $v\left(\Theta \cup\left\{\frac{1}{2} \varphi\right\}\right) \subseteq D$. 
Agora a conjunção do tipo $\alpha$, isto é, $1 \varphi \wedge \psi$. Como o conjunto $\Theta$ é satisfatível, então existe uma valoração v tal que $v(\Theta) \subseteq D$. Daí, $v(\varphi \wedge \psi)=1$ e, então, $v(\varphi)=1$ e $v(\psi)=1$. Portanto, $v(\Theta \cup\{1 \varphi, 1 \psi\}) \subseteq D$.

Para a disjunção do tipo $\alpha$ temos que $0 \varphi \vee \psi$. Como o conjunto $\Theta$ é satisfatível, então existe uma valoração v tal que $v(\Theta) \subseteq D$. Daí, $v(\varphi \vee \psi)=0$ e, então, $v(\varphi)=0$ e $v(\psi)=0$ e, portanto, $0 \varphi, 0 \psi \in D$. Assim, $v(\Theta \cup\{0 \varphi, 0 \psi\}) \subseteq D$.

Todas as regras de negação são do tipo $\alpha$.

Se temos $0 \neg \varphi$, desde que o conjunto $\Theta$ é satisfatível, então existe uma valoração $v$ tal que $v(\Theta) \subseteq D$. Daí, $v(\neg \varphi)=0$ e, então, $v(\varphi)=1$ e, portanto, $v(\Theta \cup\{1 \varphi\}) \subseteq D$.

Quando temos $\frac{1}{2} \neg \varphi$, como $\Theta$ é satisfatível, então existe uma valoração v tal que $v(\Theta) \subseteq D$. Daí, $v(\neg \varphi)=\frac{1}{2}$ e, então $v(\varphi)=\frac{1}{2}$ e, portanto, $v\left(\Theta \cup\left\{\frac{1}{2} \varphi\right\}\right) \subseteq D$.

Finalmente, se temos $1 \neg \varphi$. Como o conjunto $\Theta$ é satisfatível, então existe uma valoração $v$ tal que $v(\Theta) \subseteq D$. Daí, $v(\neg \varphi)=1$ donde segue que $v(\varphi)=0$ e, portanto, $v(\Theta \cup\{0 \varphi\}) \subseteq D$.

(ii) Para a fórmula de consistência do tipo $\beta$, temos $1 \circ \varphi$. Como $\Theta$ é satisfatível, então existe $v$ tal que $v(\Theta) \subseteq D$ e, então, $v(\circ \varphi)=1$. Consequentemente, $v(\varphi)=0$ ou $v(\varphi)=1$. Se $v(\varphi)=0$, então $v(\Theta \cup\{0 \varphi\}) \subseteq D$. Contudo, se $v(\varphi)=1$, então $v(\Theta \cup\{1 \varphi\}) \subseteq D$. De qualquer modo há um ramo tal que $v(\Theta \cup\{k \varphi\}) \subseteq D$.

Para a conjunção do tipo $\beta$, temos $0 \varphi \wedge \psi$. Como $\Theta$ é satisfatível, então existe $v$ tal que $v(\Theta) \subseteq D$. Daí, $v(\varphi \wedge \psi)=0$ e, consequentemente, $v(\varphi)=0$ ou $v(\psi)=0$. Se $v(\varphi)=0$, então $v(\Theta \cup\{0 \varphi\}) \subseteq D ;$ e se $v(\psi)=0$, então $v(\Theta \cup\{0 \psi\}) \subseteq D$.

Se temos uma disjunção do tipo $\beta$, isto é, $1 \varphi \vee \psi$, desde que $\Theta$ é satisfatível, então existe $v$ tal que $v(\Theta) \subseteq D$. Daí, $v(\varphi \vee \psi)=1$ e, consequentemente, $v(\varphi)=1$ ou $v(\psi)=1$. Se $v(\varphi)=1$, então $v(\Theta \cup\{1 \varphi\}) \subseteq D$; e se $v(\psi)=1$, então $v(\Theta \cup\{1 \psi\}) \subseteq D$.

Para $0 \varphi \rightarrow \psi$, como $\Theta$ é satisfatível, então existe $v$ tal que $v(\Theta) \subseteq D$. Daí, $v(\varphi \rightarrow \psi)=0$ e, consequentemente, $v(\psi)=0$ e $v(\varphi) \in D$. Para $v(\psi)=0$, segue que $v(\Theta \cup\{0 \psi\}) \subseteq D$. Agora, para qualquer $k \in D$, se $v(\varphi)=k$, então $v(\Theta \cup\{k \varphi\}) \subseteq D$. Logo, um dos ramos é tal que $v(\Theta \cup\{0 \psi, k \varphi\}) \subseteq D$.

(iii) Para a conjunção do tipo $\gamma$ temos $\frac{1}{2} \varphi \wedge \psi$. Como $\Theta$ é satisfatível, então existe $v$ tal que $v(\Theta) \subseteq D$. Daí, $v(\varphi \wedge \psi)=\frac{1}{2}$ e, consequentemente, $v(\varphi)=1$ e $v(\psi)=\frac{1}{2}$; ou $v(\varphi)=\frac{1}{2} e$ $v(\psi)=1$; ou $v(\varphi)=\frac{1}{2}$ e $v(\psi)=\frac{1}{2}$. Como tem que valer um destes três casos, para $k_{1}, k_{2} \in D$, segue que $v\left(\Theta \cup\left\{k_{1} \varphi, k_{2} \psi\right\}\right) \subseteq D$.

Para a disjunção do tipo $\gamma$ temos $\frac{1}{2} \varphi \vee \psi$. Como $\Theta$ é satisfatível, então existe $v$ tal que $v(\Theta) \subseteq D$. Daí, $v(\varphi \vee \psi)=\frac{1}{2}$ e, consequentemente, $v(\varphi)=0$ e $v(\psi)=\frac{1}{2} ;$ ou $v(\varphi)=\frac{1}{2}$ e $v(\psi)=0$; ou $v(\varphi)=\frac{1}{2}$ e $v(\psi)=\frac{1}{2}$. Como tem que valer um destes três casos, para $k_{1}, k_{2} \in\left\{0, \frac{1}{2}, 1\right\}$, segue que $v\left(\Theta \cup\left\{k_{1} \varphi, k_{2} \psi\right\}\right) \subseteq D$.

Para a condicional do tipo $\gamma$ temos $1 \varphi \rightarrow \psi$. Como $\Theta$ é satisfatível, então existe v tal que $v(\Theta) \subseteq D$. Daí, $v(\varphi \rightarrow \psi)=1$ e, consequentemente, $v(\varphi)=0$ ou $v(\psi)=\frac{1}{2}$ ou $v(\psi)=1$. Seja $k \in\left\{\frac{1}{2}, 1\right\}$. Se $v(\varphi)=0$, então $v(\Theta \cup\{0 \varphi\}) \subseteq D$ e se $v(\psi)=k$, então $v(\Theta \cup\{k \psi\}) \subseteq D$.

Em todos os casos, algum ramo do tableau é satisfativel.

Diante dessas definições e do Lema acima podemos provar o seguinte teorema. 
Teorema $5.8 \Gamma \Vdash \varphi \Rightarrow \Gamma \vDash \varphi$

Demonstração: Faremos a demonstração pela contra-positiva.

Se $\Gamma \not \models \varphi$, então existe uma valoração $v$, tal que $v(\Gamma) \subseteq D$ e $v(\varphi)=0$.

Seja $\Theta_{0}$ o conjunto de fórmulas sinalizadas que ocorrem no tableau inicial de $\Gamma$, de modo que $v\left(\Theta_{0}\right) \subseteq D$. Mostramos que a cada passo de expansão do tableau, sempre vai existir um ramo $\Theta_{0}$ tal que $v\left(\Theta_{0}\right) \subseteq D$.

Suponha que $v\left(\Theta_{i-1}\right) \subseteq D$. Se o ramo $\Theta_{i-1}$ for expandido por uma fórmula do tipo $\alpha$, pelo lema anterior $(i)$, temos que $v\left(\Theta_{i}\right) \subseteq D$.

No caso do ramo $\Theta_{i-1}$ ser expandido por uma fórmula do tipo $\beta$, segue pelo lema anterior (ii), que $v\left(\Theta_{i}\right) \subseteq D$.

Se o ramo $\Theta_{i-1}$ for expandido por uma fórmula do tipo $\gamma$, pelo mesmo lema item (iii) segue que $v\left(\Theta_{i}\right) \subseteq D$.

Assim, em todos os casos, temos um ramo $\Theta_{i}$ tal que $v\left(\Theta_{i}\right) \subseteq D$. Logo, sempre haverá um ramo satisfativel em $\Theta$, o qual é um conjunto saturado para baixo.

Portanto, $\Gamma \nVdash \varphi$

\section{Referências Bibliográficas}

BETH, E. W. The foundations of mathematics. Amsterdam: North Holland, 1959.

CONIGLIO, M. E.; SILVESTRINI, L. H. C. An alternative approach for quasi-truth. Logic Journal of IGPL, v. 22, n. 2, p. 387-410, 2014.

COSTA, N. C. A. da O conhecimento científico. 2a. ed. São Paulo: Discurso Editorial, 1999.

COSTA, N. C. A. da; BÉZIAU, J. Y.; BUENO, O. Elementos de teoria paraconsistente de conjuntos. Campinas: Unicamp - CLE, 1998. (Coleção CLE, v. 23)

EBBINGHAUS, H. D.; FLUM, J.; THOMAS, W. Mathematical logic. New York: SpringerVerlag, 1984.

ENDERTON, H. B. A mathematical introduction to logic. San Diego: Academic Press, 1972.

EPSTEIN, R. L. The semantic foundations of logic: propositional logics. Dordrecht: Kluwer Academic Publishers, 1990.

FEITOSA, H. A.; PAULOVICH, L. Um prelúdio à lógica. São Paulo: Editora Unesp, 2005.

FEITOSA, H. A.; SILVESTRINI, L. H. C. On the logic of pragmatic truth. In: ARENHART, J. R. B.; CONTE, J.; MORTARI, C. A. (Orgs.). Temas em filosofia contemporânea II, Florianópolis: NEL/UFSC, 2016. p. 123-136.

GENTZEN, G. Untersuchungen über das logische Schlieben. Mathematizche Zeitschrift, v. 39, 1935. 
GENTZEN, G.; SZABO, M. E. The collected papers of Gerhard Gentzen. SZABO, M. E. (Ed.). Amsterdam: North-Holland, 1969.

HINTIKKA, J. Form and content in quantification theory. Acta Philosophica Fennica v.8. 1955.

MALINOWSKI, G. Many-valued logics. Oxford: Clarendon Press, 1993.

MENDELSON, E. Introduction to mathematical logic. Princeton: D. Van Nostrand, 1964.

RASIOWA, H. An algebraic approach to non-classical logics. Amsterdam: North-Holland, 1974.

SILVA, F. S. C.; FINGER, M.; MELO, A. C. V. Lógica para computação. São Paulo: Thomson Learning, 2006.

SILVESTRINI, L. H. Uma nova abordagem para a noção de quase-verdade. 2011. $115 f$. Tese (doutorado) - Instituto de Filosofia e Ciências Humanas, Universidade Estadual de Campinas, Campinas, 2011.

SMULLYAN, R. M. First-order logic. New York: Springer-Verlag / Dover Publication, 1968.

SMUlLyAN, R. M. Lógica de Primeira Ordem. Tradução de Andréa M. A. de Campos Loparic, René Pierre Mazak e Luciano Vicente. São Paulo: Editora Unesp, 2002/2009. 